
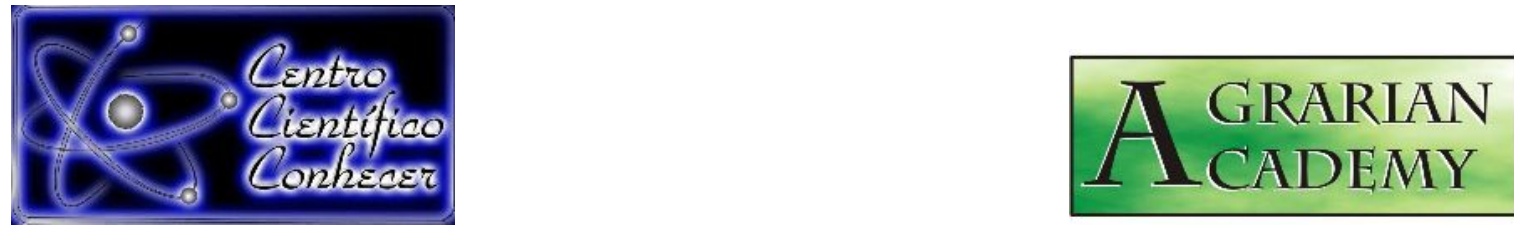

\title{
ANÁLISE DO ÍNDICE RELATIVO DE CLOROFILA EM FISÁLIS ATRAVÉS DE DIFERENTES MEDIDORES PORTÁTEIS
}

Rodrigo Ferraz Ramos ${ }^{1}$, Anderson Machado Pavanelo ${ }^{2}$, Felix Cidade do Prado ${ }^{3}$, Suzana dos Santos de Souza ${ }^{4}$, Débora Leitzke Betemps ${ }^{5}$

${ }^{1}$ Graduando em Agronomia, Laboratório de Microbiologia, na Universidade Federal da Fronteira Sul (UFFS), campus Cerro Largo, Brasil. rodrigoferrazramos@gmail.com

${ }^{2}$ Mestrando do Programa de Pós-Graduação em Ambiente e Tecnologias Sustentáveis, Laboratório de Botânica, na Universidade Federal da Fronteira Sul (UFFS), campus Cerro Largo

${ }^{3}$ Graduando em Agronomia, Laboratório de Agroecologia, na Universidade Federal da Fronteira Sul (UFFS), campus Cerro Largo

${ }^{4}$ Mestranda do Programa de Pós-Graduação em Ambiente e Tecnologias

Sustentáveis, Laboratório de Botânica, na Universidade Federal da Fronteira Sul (UFFS), campus Cerro Largo

${ }^{5}$ Professora Ajunto III, Laboratório de Agroecologia, Universidade Federal da Fronteira Sul (UFFS), campus Cerro Largo

Recebido em: 14/07/2018 - Aprovado em: 28/07/2018 - Publicado em: 31/07/2018 DOI: 10.18677/Agrarian_Academy_2018a2

\begin{abstract}
RESUMO
A análise do teor de clorofila fornece informações importantes sobre o estado fisiológico e potencial fotossintético das plantas. Devido ao fato da medição direta de clorofila ser um método destrutivo, leitores ópticos móveis são adotados enquanto ferramentas não destrutivas para a avaliação indireta dos níveis de clorofila nas plantas. A avaliação indireta da clorofila é realizada amplamente pelo SPAD-502, por apresentar valores relativos com alta correlação com os teores absolutos de clorofila. Pode-se igualmente serem adotados outros medidores portáteis, como o uso de colorímetros, que podem apresentar valores com alta correlação com os valores relativos obtidos via SPAD-502. O objetivo do presente trabalho foi avaliar a correlação entre o clorofilômetro SPAD-502 (Minolta ${ }^{\circledR}$ ) e o Colorímetro CR-400 $\left(\right.$ Minolta ${ }^{\circledR}$ ) para a análise dos índices relativos de clorofila em folhas de Physalis peruviana. Observou-se que os índices relativos de clorofila obtidos via SPAD-502 apresentam correlação altamente significativa $(p<0,01)$ com os valores de $L^{*} a^{*} b^{*}$, onue e índice chroma $\left(c^{\star}\right)$ obtidos pelo colorímetro CR-400. Os resultados obtidos indicam potencial viabilidade técnica para o uso do colorímetro CR-400 na análise indireta de clorofila em folhas de $P$. peruviana.
\end{abstract}

PALAVRAS-CHAVE: Índice de Clorofila, Physalis peruviana, SPAD-502. 


\title{
ANALYSIS OF THE RELATIVE CHLOROPHILA INDEX IN FISALS THROUGH DIFFERENT PORTABLE METERS
}

\begin{abstract}
The analysis of the chlorophyll content provides important information on the physiological state and photosynthetic potential of the plants. Because direct measurement of chlorophyll is a destructive method, mobile optical readers are adopted as non-destructive tools for the indirect evaluation of chlorophyll levels in plants. The indirect evaluation of chlorophyll is performed extensively by the SPAD502 , as it presents relative values with high correlation with the absolute chlorophyll content. Other portable meters can also be adopted, such as the use of colorimeters, which can present values with high correlation with the relative values obtained through SPAD-502. The aim of the present study was to evaluate the correlation between chlorophyllometer SPAD-502 (Minolta ${ }^{8}$ ) and Colorimeter CR-400 (Minolta $B$ ) for the analysis of relative indexes of chlorophyll in leaves of Physalis peruviana. It was observed that the relative indexes of chlorophyll obtained by SPAD502 showed a highly significant correlation $(p<0.01)$ with the values of $L^{*} a^{*} b^{*}$, ohue and chroma index $\left(\mathrm{c}^{*}\right)$ obtained by the CR-400 colorimeter. The results indicate potential technical feasibility for the use of the CR-400 colorimeter in the indirect analysis of chlorophyll in leaves of $P$. peruviana.
\end{abstract}

KEYWORDS: Chlorophyll Index, Physalis peruviana, SPAD-502.

\section{INTRODUÇÃO}

As clorofilas são os pigmentos biológicos fotossintéticos mais abundantes na Terra (KLUGE et al., 2015). A análise do teor de clorofila nas folhas é um método comumente adotado na agricultura para monitorar o desenvolvimento vegetal, fornecendo informações importantes sobre o estado fisiológico, teores de nitrogênio nas folhas e o potencial fotossintético das plantas (RICCARDI et al., 2014; YANG et al., 2015).

A medição direta de clorofila usando produtos químicos para a extração é um método destrutivo, complexo e demorado, o que levou ao desenvolvimento de leitores ópticos móveis, fornecendo ferramentas não destrutivas para a avaliação indireta dos níveis de clorofila nas plantas (CORTAZAR et al., 2015). A avaliação indireta da clorofila é realizada amplamente pelo método SPAD (Soil Plant Analysis Development) por apresentar valores relativos com alta correlação com os teores absolutos de clorofila presente nas folhas (JESUS; MARENCO, 2008). O uso do clorofilômetro SPAD-502 (Minolta ${ }^{\circledR}$, Japão) já foi relatado para diferentes culturas, como em Eucalyptus dunnii Maiden (DRANSKI et al., 2016), Lycopersicon esculentum Mill. (NOGUEIRA et al., 2018), entre outras.

Outros medidores portáteis podem ser adotados para a realização de análises não destrutivas e a determinação de índices relativos de clorofila, como o uso de colorímetros portáteis. O uso de colorímetros portáteis já foi relatado para a determinação de índices de clorofila relativa para a cultura do milho (SILVA et al., 2016), do morangueiro (TONIN et al., 2016), entre outras espécies.

Na $P$. peruviana L., análises dos índices relativos de clorofila via SPAD-502 já foram relatados, relacionando os índices relativos de clorofila das folhas com 0 estado fisiológico das plantas submetidas ao estresse hídrico (ALDANA et al., 2014), a diferentes tratamentos com nutrientes (VERMA et al., 2017), e a aplicação de diferentes fontes de nitrogênio (ANTÚNEZ-OCAMPO et al., 2016). Porém, não foi AGRARIAN ACADEMY, Centro Científico Conhecer - Goiânia, v.5, n.9; p.11 2018 
observado na literatura o uso de colorímetros portáteis para a análise de clorofila relativa em $P$. peruviana.

O objetivo do presente trabalho foi avaliar a correlação entre o clorofilômetro SPAD-502 (Minolta ${ }^{\circledR}$ ) e o Colorímetro CR-400 (Minolta ${ }^{\circledR}$ ) para a análise dos índices relativos de clorofila em Physalis peruviana.

\section{MATERIAL E MÉTODOS}

O experimento foi conduzido em solo, classificado como Latossolo Vermelho, durante os meses de setembro de 2017 a junho de 2018, na área experimental da Universidade Federal da Fronteira Sul (UFFS) campus Cerro Largo, região noroeste do Estado do Rio Grande do Sul, com clima tipo Cfa, segundo classificação de Köppen.

A Physalis peruviana L. pertence à família Solanaceae, e é conhecida popularmente como fisális. A semeadura de $P$. peruviana foi realizada em sacos plásticos com capacidade de $0,25 \mathrm{~L}$, preenchidos com substrato Carolina Soil ${ }^{\circledR}$. Após as mudas atingiram uma altura média de $15 \mathrm{~cm}$, selecionaram-se mudas plenamente saudáveis e foi realizado o transplantio das mesmas para canteiros previamente preparados, com a fertilidade e $\mathrm{pH}$ corrigido, de acordo com as exigências para produção de solanáceas (SBCS, 2004).

Para a realização das análises, realizou-se a coleta das folhas de $P$. peruviana, em diferentes ramos laterais, quando as plantas atingiram o Estádio Fenológico 8, correspondente ao período de colheita (BETEMPS et al., 2014). Com o intuito de obter um gradiente para a análise relativa dos teores de clorofila, foram selecionadas folhas com diferentes graus de senescência, estabelecendo-se arbitrariamente oito estádios de senescência das folhas (Figura 1), correspondente as diferenças visuais na coloração das folhas.

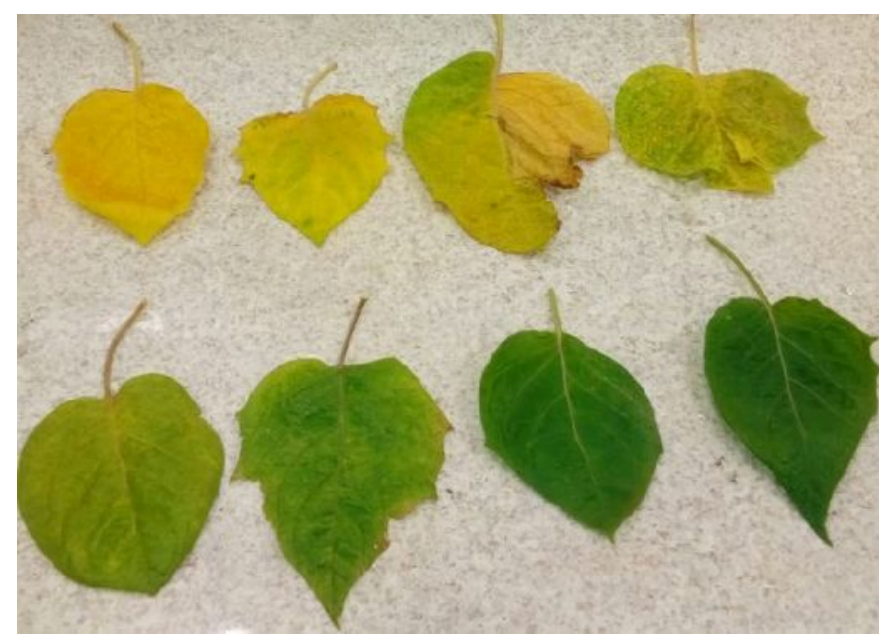

FIGURA 1 - Diferentes estádios visuais de senescência em folhas de Physalis peruviana.

Fonte: Elaborado pelos autores.

Para cada estádio de senescência estabelecido, foram selecionadas três folhas visualmente semelhantes. Para cada folha selecionada, foram realizadas na parte adaxial duas leituras, uma leitura de cada lado da nervura central, do índice de clorofila $a(\mathrm{Chl} a)$, clorofila $b(\mathrm{Chl} b)$ e clorofila $a+b(\mathrm{Chl}$ total), por meio do medidor indireto de clorofila SPAD-502 (Soil Plant Analysis Development, Minolta ${ }^{\circledR}$, Japão).

Para as mesmas folhas, realizou-se a análise da coloração na parte adaxial, através de duas leituras, uma leitura de cada lado da nervura central, por meio do 
Colorímetro CR-400 (Minolta ${ }^{\circledR}$ ). Através do padrão CIE-Lab, obtiveram-se os valores dos componentes $L^{*}, a^{*}$ e $b^{*}$. Os valores obtidos foram expressos em Ângulo Hue (hue) através da fórmula [arco tangente $\left.\left(\mathrm{b}^{*} / \mathrm{a}^{*}\right)\right]$, e expressos em índice Chroma $\left(\mathrm{c}^{*}\right)$ através da fórmula $\left[\left(a^{* 2}+b^{* 2}\right)^{1 / 2}\right]$ (SÁ et al., 2008).

Os valores de $L^{*}$ variam do claro ao escuro, sendo o valor 100, correspondente à cor branca e o valor 0 (zero) à cor preta; o componente $a^{*}$ varia entre o vermelho e o verde, os valores positivos correspondem ao vermelho, o 0 (zero) ao cinza e, os valores negativos, à cor verde; e o componente $b^{*}$ varia do azul ao amarelo, os valores negativos correspondem ao azul, o 0 (zero) ao cinza e, os valores positivos, à cor amarela (CONTI et al., 2002). Os valores dos componentes $L^{*} a^{*} b^{*}$ foram denominados no presente estudo de índice $L^{*}$, índice $a^{*} e$ índice $b^{*}$, respectivamente.

Os índices relativos de clorofila ( $\mathrm{Chl} a, \mathrm{Chl} b$ e $\mathrm{Chl}$ total) obtidos pelo SPAD502 foram relacionados com os diferentes índices $\left(L^{*}, a^{*}, b^{*}\right.$, ohue e $\left.c^{*}\right)$ obtidos pelo colorímetro CR-400 mediante análise de regressão, as equações foram obtidas pelo software Excel ${ }^{\circledR}$. Os índices relativos de clorofila do SPAD-502 foram considerados as variáveis dependentes $(\mathrm{y})$ e os valores obtidos pelo CR-400 como variáveis independentes (x), como proposto por Silva et al. (2016).

Posteriormente, os resultados obtidos foram submetidos à análise de correlação, determinando-se o coeficiente de correlação de Pearson, através do Software R Studio (R CORE TEAM, 2018). O coeficiente de correlação Pearson ( $r$ ) varia de -1 a 1 , onde o sinal indica direção positiva ou negativa do relacionamento $\mathrm{e}$ o valor sugere a força da relação entre as variáveis, sendo que uma correlação perfeita (-1 ou 1) indica que o escore de uma variável pode ser determinado exatamente ao se saber o escore da outra (FIGUEIREDO FILHO; SILVA JÚNIOR, 2009).

\section{RESULTADOS E DISCUSSÃO}

Na Figura 2 encontra-se plotado os resultados, equações e coeficientes de determinação $\left(R^{2}\right)$ para as diferentes variáveis analisadas, enquanto que na Tabela 1 encontram-se os coeficientes de correlação de Pearson ( $r$ ) para as diferentes variáveis. No presente estudo, observou-se que todas as variáveis apresentaram correlação $(r)$ altamente significativa $(p<0,01)$.

Para as variáveis onue (' $x$ '), obtidas pelo colorímetro CR-400, e o índice de clorofila a ('y') obtido pelo SPAD-502, obteve-se um coeficiente de determinação de 0,9157 (Figura 1A), enquanto que para as variáveis onue (' $x$ ') e $o$ índice de clorofila $b$ (' $y$ ') obteve menor coeficiente de determinação $\left(R^{2}=0,7885\right.$, Figura 1B), indicando que o índice de $\mathrm{Chl}$ a ajusta-se mais adequadamente com $\mathrm{o}$ hue.

Para ambas as situações supracitadas, a correlação $(r)$ foi positiva e altamente significativa $(p<0,01)$, ou seja, à medida que os valores obtidos para o ohue (colorímetro) aumentam, os valores obtidos pelo SPAD-502 aumentam para os índices de $\mathrm{Chl} a$ e Chl $b$, e vice-e-versa. Resultados intermediários foram observados para as variáveis ${ }^{\circ}$ hue (' $x$ ') e o índice de clorofila total (' $y$ ') (resultados não plotados), foi obtido um coeficiente de determinação de 0,8981 e a seguinte equação linear: $y=0,725 x-54,596$.

Resultados semelhantes foram observados para a cultura do milho (AMARANTE et al., 2010) e para o morangueiro (TONIN et al., 2016), sendo que ambos os autores observaram aumento nos valores do ohue, obtidos pelo colorímetro CR-400 (Minolta ${ }^{\circledR}$ ), a medida que os teores de clorofila (via método destrutivo) aumentavam nas folhas. 
Para as variáveis índice $b^{*}$ (Figura 1C, ' $x$ ') e índice $a^{*}$ (Figura 1D, ' $x$ ') obtidos pelo colorímetro, e o índice de clorofila total (' $y$ '), obteve-se um $R^{2}$ de 0,9214 e 0,6326 , respectivamente. $\mathrm{Na}$ análise de correlação de Pearson, para ambas as variáveis foram obtidas correlação altamente significativa $(p<0,01)$ e negativa, ou seja, a medida que os valores de Chl Total aumentam, os valores de $a^{*}$ e $b^{*}$ diminuem. Contudo, a ocorrência de um baixo $R^{2}(0,6326)$ entre o índice $a^{*}$ (Figura 1C) e o índice de Chl Total, indica baixa confiança entre as leituras obtidas pelo colorímetro e o SPAD-502 para essas duas variáveis, quando correlacionadas.

De acordo com os resultados supracitados, à medida que os índices de clorofila obtidos via SPAD-502 aumentam, os valores obtidos para o componente a $^{*}$ diminuem em direção ao espaço de cor verde, indicando um enverdecimento das folhas; enquanto que a medida que os índices de clorofila via SPAD-502 diminuem, os valores correspondentes ao componente $b^{*}$ aumentam em direção ao espaço de cor amarela, indicando aumento da clorose nas folhas (amarelecimento).

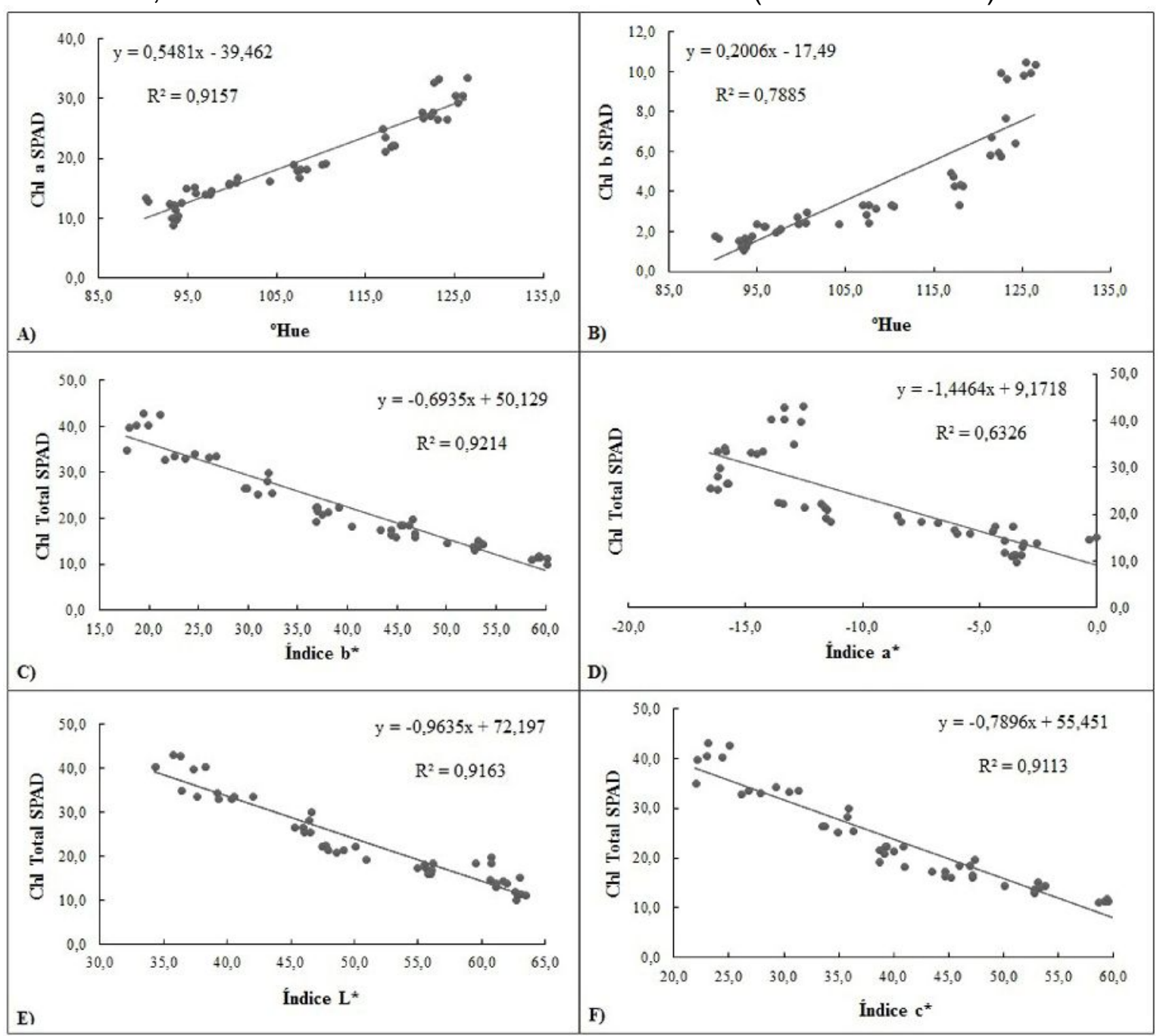

FIGURA 2 - Análises das relações entre os índices relativos de clorofila através do SPAD502 e o colorímetro CR-400 em folhas de Physalis peruviana. A) Ângulo Hue (ํhue, ' $x$ ') e Î́ndice de Clorofila $a$ (Chl a, ' $y$ '); B) Ângulo Hue (ํhue, ' $x$ ') e Índice de Clorofila $b$ (Chl b, ' $y$ '); C) Componente $b^{*}$ (Índice b*, ' $x$ ') e Îndice de Clorofila $a+b$ (Chl Total, ' $y$ '); D) Componente $a^{*}$ (Índice $a^{*},{ }^{\prime} x$ ') e Índice de Clorofila $a+$ $b$ (Chl Total, 'y'); E) Componente $L^{*}$ (Índice L*, ' $x$ ') e Clorofila $a+b$ (Chl Total, 'y'); F) Componente $c^{*}$ (Índice $c^{*}, x^{\prime}$ ') e Clorofila $a+b$ (Chl Total, ' $y$ '). 
No presente estudo, para a variável índice L* (Figura 1E, ' $x$ ') e Chl Total ('y') obteve-se um coeficiente de determinação de 0,9163 , sendo a correlação altamente significativa $(p<0,01)$ e negativa, ou seja, a medida que os valores obtidos pelo colorímetro para $o$ índice $L^{*}$ aumentam, os valores para o índice de clorofila total, obtidos via SPAD-502, decrescem. Resultados semelhantes foram obtidos para o índice $C^{*}$ (Figura 1F, ' $x$ ') em relação ao índice de Chl Total (' $y$ '), que apresentou um $R^{2} \quad 0,9113$, e correlação $(r)$ altamente significa $(p<0,01)$ e negativa. Esses resultados indicam uma diminuição do brilho e na cromaticidade das folhas à medida que os teores relativos de clorofila aumentam.

TABELA 1 - Análise do coeficiente de correlação de Pearson ( $r$ ) entre as variáveis: índice de clorofila $a(\mathrm{Chl} a)$, índice de clorofila $b(\mathrm{Chl} b)$, índice de clorofila $a+b$ (Chl total) mensurados através do medidor indireto de clorofila SPAD-502, e os índices $L^{*}, a^{*}, b^{*}, c^{*}$ e ângulo Hue (o Hue) mensurados através do Colorímetro CR-400 (Minolta ${ }^{\circledR}$ ).

\begin{tabular}{|c|c|c|c|c|c|c|c|}
\hline & Chl a & Chl $b$ & Chl total & $L^{*}$ & $a^{*}$ & $b^{*}$ & $\mathrm{C}^{*}$ \\
\hline Chl $b$ & $0,8575^{\prime \prime}$ & & & & & & \\
\hline Chl total & $0,9895^{* *}$ & $0,9228^{\star *}$ & & & & & \\
\hline$L^{*}$ & $-0,9634^{* *}$ & $-0,8402^{* *}$ & $-0,9573^{* *}$ & & & & \\
\hline$a^{*}$ & $-0,8143^{* *}$ & $-0,6613^{* *}$ & $-0,7954^{* *}$ & $0,8554^{\star *}$ & & & \\
\hline$b^{*}$ & $-0,9707^{* *}$ & $-0,8300^{* *}$ & $-0,9599^{* *}$ & $0,9803^{* *}$ & $0,8544^{* *}$ & & \\
\hline$c^{*}$ & $-0,9655^{* *}$ & $-0,8254^{* *}$ & $-0,9546^{\star *}$ & $0,9744^{\star \star}$ & $0,8311^{\star *}$ & $0,9983^{* *}$ & \\
\hline ohue & $0,9569^{* *}$ & $0,8235^{* *}$ & $0,9477^{* *}$ & $-0,9677^{* *}$ & $-0,9235^{* *}$ & $-0,9612^{* *}$ & $-0,9448^{* *}$ \\
\hline
\end{tabular}

${ }^{\star *} p$-value $<0,01$

Corroborando com esses resultados, Amarante et al. (2010) observaram decréscimo nos valores de $\mathrm{L}^{*}$ e $\mathrm{C}^{*}$, obtidos pelo colorímetro CR-400 (Minolta ${ }^{\circledR}$ ), com o incremento nos teores de clorofila via método destrutivo em folhas de milho (Zea mays). Contrariamente esses resultados, Silva et al. (2016) observaram correlação positiva e significativa entre os índices de clorofila total obtidos pelo SPAD-502 e o índice $L^{*}$ obtido pelo colorímetro Meter-10 $\left(\right.$ Minolta $\left.^{\circledR}\right)$ para a cultura do milho.

De acordo com os resultados, observou-se que os valores via sistema CIE L*a*b* obtidos através do colorímetro CR-400, apresentam alta correlação com os índices relativos de clorofila obtidos via SPAD-502, indicando potencial viabilidade técnica do uso do colorímetro para análise indireta de clorofila em folhas de $P$. peruviana.

Contudo, ressalta-se que para a adoção do colorímetro CR-400 para análise de clorofila indireta, é necessária a realização da calibração do colorímetro com as extrações direta de clorofila (AMARANTE et al., 2010). Assim, indica-se a necessidade da realização da calibração dos índices relativos de clorofila via SPAD502 e o colorímetro CR-400 com os teores de clorofila via extração destrutiva, que até o momento não foi relatada na literatura científica para a $P$. peruviana.

\section{CONCLUSÃO}

Conclui-se que os índices relativos de clorofila obtidos via SPAD-502 $\left(\right.$ Minolta $\left.^{\circledR}\right)$ apresentam alta correlação com os valores obtidos pelo colorímetro CR400 (Minolta $^{\circledR}$ ), indicando potencial viabilidade técnica para o uso do colorímetro na análise indireta de clorofila em folhas de $P$. peruviana. 


\section{REFERÊNCIAS}

ALDANA, F.; GARCÍA, P. N.; FISCHER, G. Effect of waterlogging stress on the growth, development and symptomatology of cape gooseberry (Physalis peruviana L.) plants. Revista de la Academia Colombiana de Ciencias Exactas, Físicas y Naturales, v. 38, n. 149, p. 393-400, 2014. Disponível em: <http://www.scielo.org.co/pdf/racefn/v38n149/v38n149a05.pdf>.

AMARANTE, C. V. T.; STEFFENS, C. A.; SANGOI, L.; ZANARDI, O. Z.; MIQUELOTO, A.; SCHWEITZER, C. Quantificação de clorofilas em folhas de milho através de métodos ópticos não destrutivos. Revista Brasileira de Milho e Sorgo, v. 9, n. 1, p. 39-50, 2010. Disponível em: <http://dx.doi.org/10.18512/19806477/rbms.v9n1p39-50 >. DOI: 10.18512/1980-6477/rbms.v9n1p39-50

ANTÚNEZ-OCAMPO, O. M.; SANDOVAL-VILLA, M.; ALCÁNTAR-GONZÁLEZ, G.; ALVARADO-LÓPEZ, J.; SABINO-LÓPEZ, J. E. Floración y frutificación de Physalis peruviana L. por la aplicación de amônio y nitrato, edad y vigor de la planta. Agrociencia, v. 50, n. 5, p. 603-615, 2016. Disponível em: <http://www.scielo.org.mx/pdf/agro/v50n5/1405-3195-agro-50-05-603.pdf>.

BETEMPS, D. L.; FACHINELLO, J. C.; LIMA, C. S. M.; GALARÇA, S. P.; RUFATO, A. R. Época de semeadura, fenologia e crescimento de plantas de fisális no sul do Brasil. Revista Brasileira de Fruticultura, v. 36, n. 1, p. 179-185, 2014. Disponível em: <http://dx.doi.org/10.1590/0100-2945-292/13>. DOI: 10.1590/0100-2945-292/13

CONTI, J. H.; MINAMI, K.; TAVARES, F. C. A. Produção e qualidade de frutos de diferentes cultivares de morangueiro em ensaios conduzidos em Atibaia $e$ Piracicaba. Horticultura Brasileira, v. 20, n. 1, p. 10-17, 2002. Disponível em: <http://dx.doi.org/10.1590/S0102-05362002000100002>. DOI: $10.1590 / S 0102-$ 05362002000100002.

CORTAZAR, B.; KOYDEMIR, H. C.; TSENG, D.; FENG, S.; OZCAN, A. Quantification of plant chlorophyll content using google glass. Lab on a Chip, v. 15, n. $7, \quad$ p. $\quad 1-9, \quad 2015 . \quad$ Disponível em: <https://www.ncbi.nlm.nih.gov/pmc/articles/PMC4366296/>. $10.1039 / \mathrm{c} 4 \mathrm{lc} 01279 \mathrm{~h}$

DRANSKI, J. A. L.; MALAVASI, U. C.; MALAVASI, M. M. Estimating chlorophyll content from Eucalyptus dunnii leaves by reflectance values. Semina: Ciências Agrárias, v. 37, n. 3, p. 1277-1283, 2016. Disponível em: <http://www.redalyc.org/pdf/4457/445746397047.pdf>. DOI: 10.5433/16790359.2016v37n3p1277

FIGUEIREDO FILHO, D. B. ; SILVA JÚNIOR, J. A. ; Desvendando os mistérios do coeficiente de correlação de Pearson (r). Revista Política Hoje, v. 18, n. 1, p. 115146, $2009 . \quad$ Disponível $<$ https://periodicos.ufpe.br/revistas/politicahoje/article/viewFile/3852/3156>.

JESUS, S. V.; MARENCO, R. A. O SPAD-502 como alternativa para a determinação dos teores de clorofila em espécies frutíferas. Acta Amazonica, v. 38, n. 4, p. 815- 
818, 2008. Disponível em: <http://dx.doi.org/10.1590/S0044-59672008000400029>. DOI: $10.1590 /$ S0044-59672008000400029

KLUGE, R. A.; TEZOTTO-ULIANA, J. V.; SILVA, P. P. M. Aspectos fisiológicos e ambientais da fotossíntese. Revista Virtual de Química, v. 7, n. 1, p. 56-73, 2015. Disponível em: <http://rvq.sbq.org.br/imagebank/pdf/v7n1a04.pdf>. DOI: $10.5935 / 1984-6835.20150004$

NOGUEIRA, B. B.; IGLESIAS, L.; MESQUITA, J. V.; NAKATANI, M. C.; PUTTI, F. F. Índice SPAD em plantas de tomateiro cultivado em fibra de coco e submetido a pulsos de fertirrigação. Brazilian Journal of Biosystems Enginnering, v. 12, n. 1, p. 1-6, 2018. Disponível em: <http://dx.doi.org/10.18011/bioeng2018v12n1p1-6>. DOI: 10.18011/bioeng2018v12n1p1-6

R CORE TEAM. R: A language and environment for statistical computing. $R$ Foundation for Statistical Computing, Vienna. 2018.

RICCARDI, M.; MELE, G.; PULVENTO, C.; LAVINI, A.; D'ANDRIA, R.; JACOBSEN, $\mathrm{S}$. E. Non-destructive evaluation of chlorophyll content in quinoa and amaranth leaves by simple and multiple regression analysis of RGB image components. Photosynth Res, v. 120, n. 3, p. 263-272, 2014. Disponível em: <https://doi.org/10.1007/s11120-014-9970-2>. DOI: 10.1007/s11120-014-9970-2

SÁ, C. R. L.; SILVA, E. O.; TERAO, D.; OSTER, A. H. Efeito do $\mathrm{KMnO}_{4}$ e 1-MCP com atmosfera controlada na conservação pós-colheita de melão Cantaloupe. Revista Ciência Agronômica, v. 39, n. 01, p. 60-69, 2008. Disponível em: http://www.ceinfo.cnpat.embrapa.br/arquivos/artigo_3300.pdf

SBCS, Sociedade Brasileira de Ciência do Solo. Manual de adubação e de calagem para os Estados do Rio Grande do Sul e de Santa Catarina. Comissão de Química e Fertilidade do Solo. 10ª ed. Porto Alegre: SBCS, 2004. 400 p.

SILVA, T. R. B.; FELIX, J. C.; MIGLIAVACCA, R. A.; KOHATSU, D. S. Utilização do colorímetro como medidor portátil de clorofila em folhas de milho. Ciência, Tecnologia \& Ambiente, v. 4, n. 1, p. 1-4, 2016. Disponível em: <http://dx.doi.org/10.4322/2359-6643.04101>. DOI: 10.4322/2359-6643.04101

TONIN, J.; MACHADO, J. T. M.; ROHRIG, B.; SOBUCKI, L.; RICHTER, A. F.; BETEMPS, D. L.; SCHMITT, O. J.; SCHINEIDER, E. P. Modelos lineares e nãolineares para determinação indireta de clorofila em folhas de morangueiro. Revista Interdisciplinar de Ensino, Pesquisa e Extensão, v. 3, n. 1, p. 216-223, 2016. Disponível $<$ http://revistaeletronica.unicruz.edu.br/index.php/eletronica/article/view/216223/pdf_49>.

VERMA, A.; SINGH, S. P.; SINGH, R. K.; SINGH, B, K. Response of chlorophyll and nutrients concentration in leaves of cape gooseberry (Physalis peruviana L.) to integrated nutrient management. International Journal of Current Microbiology 
and Applied Sciences, v. 6, n. 9, p. 2647-2651, 2017. Disponível em: <https://doi.org/10.20546/ijcmas.2017.609.326>. DOI: 10.20546/ijcmas.2017.609.326

YANG, H.; YANG, J.; LV, Y.; HE, J. SPAD values and nitrogen nutrition index for evaluation of rice nitrogen status. Plant Production Science, v. 17, n. 1, p. 81-92, 2015. Disponível em: <https://doi.org/10.1626/pps.17.81>. DOI: 10.1626/pps.17.81 